\title{
Improving Solubility and Bioavailability of Breviscapine with Mesoporous Silica Nanoparticles Prepared Using Ultrasound-Assisted Solution-Enhanced Dispersion by Supercritical Fluids Method
}

This article was published in the following Dove Press journal:

International Journal of Nanomedicine

\section{Gang Yang* \\ Zhe Li* \\ Feihua Wu \\ Minyan Chen \\ Rong Wang \\ Hao Zhu \\ Qin Li \\ Yongfang Yuan}

Department of Pharmacy, Shanghai 9th People's Hospital, Shanghai Jiao Tong University School of Medicine, Shanghai 2000 II, People's Republic of China

*These authors contributed equally to this work
Correspondence: Yongfang Yuan Department of Pharmacy, Shanghai 9th People's Hospital, Shanghai Jiao Tong University School of Medicine, Shanghai 2000 I I, People's Republic of China $\mathrm{Tel} / \mathrm{Fax}+86-2 \mathrm{I}-56786907$

Email nmxyyf@I26.com
Background: Breviscapine (BRE) has significant efficacy in cardiovascular disease, but the poor water solubility of breviscapine affects its oral absorption and limits its clinical application. In this study, supercritical carbon dioxide $\left(\mathrm{SCF}-\mathrm{CO}_{2}\right)$ technology was used to improve the solubility and bioavailability of BRE loaded into mesoporous silica nanoparticles (MSNs).

Methods: The solubility of BRE in $\mathrm{SCF}-\mathrm{CO}_{2}$ was measured under various conditions to investigate the feasibility of preparing drug-loaded MSNs by using ultrasound-assisted solution-enhanced dispersion by supercritical fluids (USEDS). The preparation process of drugloaded MSNs was optimized using the central composite design (CCD), and the optimized preparation was comprehensively characterized. Furthermore, the drug-loaded MSNs prepared by the conventional method were compared. Finally, the dissolution and bioavailability of the preparations were evaluated by in vitro release and pharmacokinetics study.

Results: The solubility of $\mathrm{BRE}$ in $\mathrm{SCF}-\mathrm{CO}_{2}$ was extremely low which was suitable to prepare BRE-loaded MSNs by USEDS technology. The particle size of the preparation was $177.24 \mathrm{~nm}$, the drug loading was $8.63 \%$, and the specific surface area was $456.3 \mathrm{~m}^{2} / \mathrm{g}$. As compared to the conventional preparation method of solution impregnation-evaporation (SIV), the formulation prepared by USEDS technology has smaller particle size, higher drug loading, less residual solvent and better stability. The results of the in vitro release study showed that drug-loaded MSNs could significantly improve drug dissolution. The results of pharmacokinetics showed that the bioavailability of drug-loaded MSNs was increased 1.96 times compared to that of the BRE powder.

Conclusion: Drug-loaded MSNs can significantly improve the solubility and bioavailability of BRE, indicating a good application prospect for MSNs in improving the oral absorption of drugs. In addition, as a solid dispersion preparation technology, USEDS technology has incomparable advantages.

Keywords: greviscapine, mesoporous silica nanoparticles, supercritical carbon dioxide technology, solubility, bioavailability

\section{Introduction}

Breviscapine (BRE), a natural flavonoid derived from the Chinese herb Erigeron breviscapus (Vant.) Hand-Mazz, exhibits various biological and pharmacological effects. ${ }^{1}$ It was widely used in the clinic for the treatment of cardiovascular and cerebrovascular diseases, such as hypertension, cerebral infarction, angina pectoris 
and coronary heart disease. ${ }^{2-4}$ Scutellarin is the major active component of BRE that is responsible for its pharmacological activity. However, despite these pharmacological functions, BRE also has some disadvantageous characteristics such as low aqueous solubility, poor chemical stability, short biological half-life and unsatisfactory bioavailability, which greatly limit its clinical application. ${ }^{5,6}$ Therefore, it is necessary to develop a formulation that can overcome the above shortcomings.

Various methods have been developed to solve the formulation challenges of insoluble drugs, such as ultrafine grinding, ${ }^{7}$ nanocrystal techniques, ${ }^{8}$ lipid-based formulation, ${ }^{9}$ solid dispersions ${ }^{10}$ and so on. With the development of nanotechnology, mesoporous silica nanoparticles (MSNs) have attracted wide attention for their potential in biomedical applications. MSNs have many excellent characteristics, such as large surface area, tunable pore size, high stability, excellent biocompatibility, and in vivo biodegradability. ${ }^{11-14} \mathrm{MSNs}$ have been regarded as the next-generation pharmaceutical carrier due to their ability to enhance the efficacy of drugs by improving their aqueous solubility and enhancing bioavailability. The ability of MSNs to enhance the solubility and dissolution rate of drugs is due to their small nanopores, which can convert a crystalline drug into an amorphous form. Furthermore, the wettability and porosity of hydrophobic drugs loaded in ordered MSNs can be improved. ${ }^{15}$

Several methods have been used to load drugs into MSNs such as dropwise addition method, ${ }^{16}$ hot melting method $^{17}$ and solution impregnation-evaporation (SIV) method. ${ }^{18}$ Among them, SIV method is most commonly used. However, all these methods use solvent and heating-based approaches. The use of organic solvents often results in high solvent residues which could cause undesirable effects on the human body and environment. Moreover, it is reported that when highly polar solvents are used, the amount of adsorbed drug is limited. ${ }^{19}$ The effect of the solvent on drug infiltration is due to the competition between drug and solvent during the adsorption process. ${ }^{20,21}$ In the melting method, drug inevitably need to be heated to a high temperature. This could lead to thermal degradation of the bioactive compound, which does not apply to heat-sensitive drugs. ${ }^{22}$

In recent years, supercritical fluid of carbon dioxide $\left(\mathrm{SCF}-\mathrm{CO}_{2}\right)$ technology with superior properties has presented an alternative and interesting route for drug loading in nano-delivery systems. $\mathrm{SCF}-\mathrm{CO}_{2}$ has an excellent solvation effect based on its lower viscosity and higher diffusivity. Commonly used $\mathrm{SCF}-\mathrm{CO}_{2}$ is chemically quite inert, economical, safe and easily available. ${ }^{23-25}$ In addition, it can be used to process thermolabile drugs due to its mild critical temperature $\left(31.06{ }^{\circ} \mathrm{C}\right)$ and critical pressure (7.39 MPa). The main supercritical fluid techniques are known as the rapid expansion of supercritical solutions (RESS), ${ }^{26}$ particles from gassaturated solutions (PGSS), ${ }^{27}$ gas anti-solvent technique (GAS), ${ }^{28}$ supercritical anti-solvent technique $(\mathrm{SAS})^{29}$ and solution-enhanced dispersion by supercritical fluids (SEDS). ${ }^{30}$ GAS, SAS and SEDS are based on the theory that SCF can be dissolved in a solvent, but the solute must be sparingly soluble in the SCF. The solute would achieve a great and fast supersaturated state and form small particles while the solution would rapidly be dispersed in the SCF. ${ }^{31}$ SEDS, in particular, has advantages in drug loading. This semi-continuous method involves atomizing the solution into a supercritical atmosphere. However, unlike other main SCF techniques, the nozzle of SEDS presents a coaxial design with a mixing length, in order to produce super-saturated solutions, facilitate precipitation of the solid in the form of small particles with high dispersion. ${ }^{32}$ In addition, we added an ultrasonic system to enhance the effect of SEDS technology which can obtain further smaller particles and make the drug more evenly loaded into the carriers.

In the present study, BRE solid dispersion with MSNs as the carrier was prepared to enhance the dissolution and oral bioavailability of BRE by the ultrasound-assisted SEDS method (USEDS). Comparisons were made between BREloaded MSNs (BRE-MSNs) prepared by a conventional method (SIV method) and the USEDS method. Samples of the BRE-MSNs were characterized in terms of their mean particle size, morphology, specific surface area, drug loading, physical state, and residual solvent. Furthermore, the intestinal toxicity was determined by a cell viability experiment. Moreover, the drug release and in vivo pharmacokinetic of BRE-MSNs were investigated in detail.

\section{Materials and Methods Materials}

BRE (purity $>96 \%$ ) was purchased from Chuxiong Yunzhi Phytopharmaceutical Co., Ltd. (Chuxiong, People's Republic of China). MSNs were synthesized according to the method developed by Tripathi et al. ${ }^{33} \mathrm{CO}_{2}$ with a purity of $99.99 \%$ was provided by Shanghai Jiao Tong University (Shanghai, People's Republic of China). All other chemicals were obtained from Sinopharm Chemical 
Reagent (Shanghai, People's Republic of China) and were of high-performance liquid chromatography (HPLC) or analytical grade.

\section{Animals and Cell Lines}

Animal studies with male Wistar rats weighing $250 \pm 10 \mathrm{~g}$ were conducted in accordance with the Guiding Principles in the Care and Use of Laboratory Animals published by the US National Institutes of Health, and the ethical approval was obtained from the ethics committee of Ninth People's Hospital, affiliated with Shanghai Jiao Tong University School of Medicine before the study. All the animals were provided by the university's Laboratory Animal Center and were maintained at the hospital's Animal Research Center. The animals were housed in standard cages at $25 \pm 2{ }^{\circ} \mathrm{C}$ and 70 $\pm 5 \%$ relative humidity under natural conditions and were provided access to food and water libitum before the start of the study. Caco-2 cells were supplied by the Chinese Academy of Medical Sciences (Beijing, China).

\section{High-Performance Liquid Chromatography (HPLC) Analysis}

BRE quantitative determinations were performed by using the HP 1260 HPLC system (Agilent Technologies, Inc., Santa Clara, CA, USA) with a reverse-phase Diamonsil ${ }^{\circledR}$ Plus C18 column, $(250 \mathrm{~mm} \times 4.6 \mathrm{~mm}, 5 \mu \mathrm{m}$; Dikma Technologies, Foothill Range, CA, USA). The mobile phase consisted of methanol, acetonitrile and $20 \mathrm{mmol} / \mathrm{L}$ phosphate buffer $(17: 17: 66, \mathrm{v} / \mathrm{v})$ at a flow rate of $0.8 \mathrm{~mL} /$ min. The column temperature was maintained at $25^{\circ} \mathrm{C}$ and the effluent was monitored at $334 \mathrm{~nm}$. Quantified samples were filtered through a $0.45-\mu \mathrm{m}$ filter membrane prior to automatic injection into the HPLC system.

\section{Solubility Determination}

The solubility of BRE in $\mathrm{SCF}-\mathrm{CO}_{2}$ is an important parameter for the preparation process of USEDS. Thus, the solubility of BRE in SCF- $\mathrm{CO}_{2}$ was measured in a semidynamic apparatus (Figure 1A) following to our previous report. ${ }^{34}$ In the present study, the solubility of BRE in SCF$\mathrm{CO}_{2}$ under a pressure of 8-22 $\mathrm{MPa}$ and a temperature of 308-338 K was measured. Approximately $200 \mathrm{mg}$ of BRE was packed into the saturation cell and the measurement procedure used in this study was as follows: In the beginning, $\mathrm{CO}_{2}$ was entered by a high-pressure syringe pump into the saturation cell to remove the air by adjusting the pressure relief valve 6. Liquid $\mathrm{CO}_{2}$ was compressed and passed into the vessel, then heated to the desired temperature. The temperature of system was controlled by temperature sensor with a precision of $1 \mathrm{~K}$. After increasing the pressure of $\mathrm{CO}_{2}$ to the desired value, valves 1 and 6 were closed. The SCF$\mathrm{CO}_{2}$ in the system was circulated by a circulation pump. During the experiments, BRE powder was dissolved at a constant desired condition in contact with $\mathrm{SCF}-\mathrm{CO}_{2}$ for about $90 \mathrm{~min}$ to guarantee the attainment of solubility equilibrium. Then, the inlet valves $(2,3)$ and outlet the valves $(4,5)$ of the U-sample collection tube with dissolved $\mathrm{BRE}$ were closed. The mass of SCF- $\mathrm{CO}_{2}$ can be calculated by the $\mathrm{CO}_{2}$ density under specific conditions and the volume of U-sample collection. Finally, the U-sample collection tube was brought down, cooled, and depressurized using a beaker filled with $20 \mathrm{~mL}$ ethanol to dissolve the drug in the collection tube. The collection tube was then rinsed three times with ethanol, and the washing solvent was combined with the beaker for BRE analyses by HPLC. All the experiments were performed in triplicates.

\section{Preparation of BRE-MSNs}

The experimental device was provided by Nantong Huaxing Petroleum Devices Co., Ltd. (Nantong, China), and is shown schematically in Figure 1B. Briefly, the USEDS apparatus consisted of three main components: a $\mathrm{CO}_{2}$ conveying system, a solution delivery system, and a precipitation system. $\mathrm{SCF}-\mathrm{CO}_{2}$ and the drug solution were pumped into the view vessel through different pipes of coaxial nozzles (inner diameter $0.2 \mathrm{~mm}$, outer diameter $1 \mathrm{~mm}$ ) and discharged continuously from the bottom. In the USEDS method, a fixed quantity of MSNs was placed in the view vessel. Then, $\mathrm{CO}_{2}$ from the cylinder was cooled down by the refrigerator and compressed into the view vessel by a high-pressure pump. The system was boosted to the desired pressure by injection of $\mathrm{CO}_{2}$ and then preheated to the desired temperature regulated by a thermostat. Valve $\mathrm{C}$ was adjusted to keep the pressure stable in the vessel. After the conditions reached the set values, the ultrasonic rod started to vibrate. Then, a certain amount of BRE was dissolved in a certain volume of methanol. The solution was delivered into the view vessel through the coaxial nozzle by a high-pressure constant flow pump (11) (LC100; Nantong, People's Republic of China) at a constant flow rate. When the solution was sprayed into the view vessel filled with $\mathrm{SCF}-\mathrm{CO}_{2}$, a rapid mutual diffusion at the interface between the SCF- $\mathrm{CO}_{2}$ and the solution occurred instantaneously. Then, the drug precipitated and got highly dispersed into the MSNs under the action of rapid solvent extraction with $\mathrm{CO}_{2}$ and ultrasonic agitation. When the 

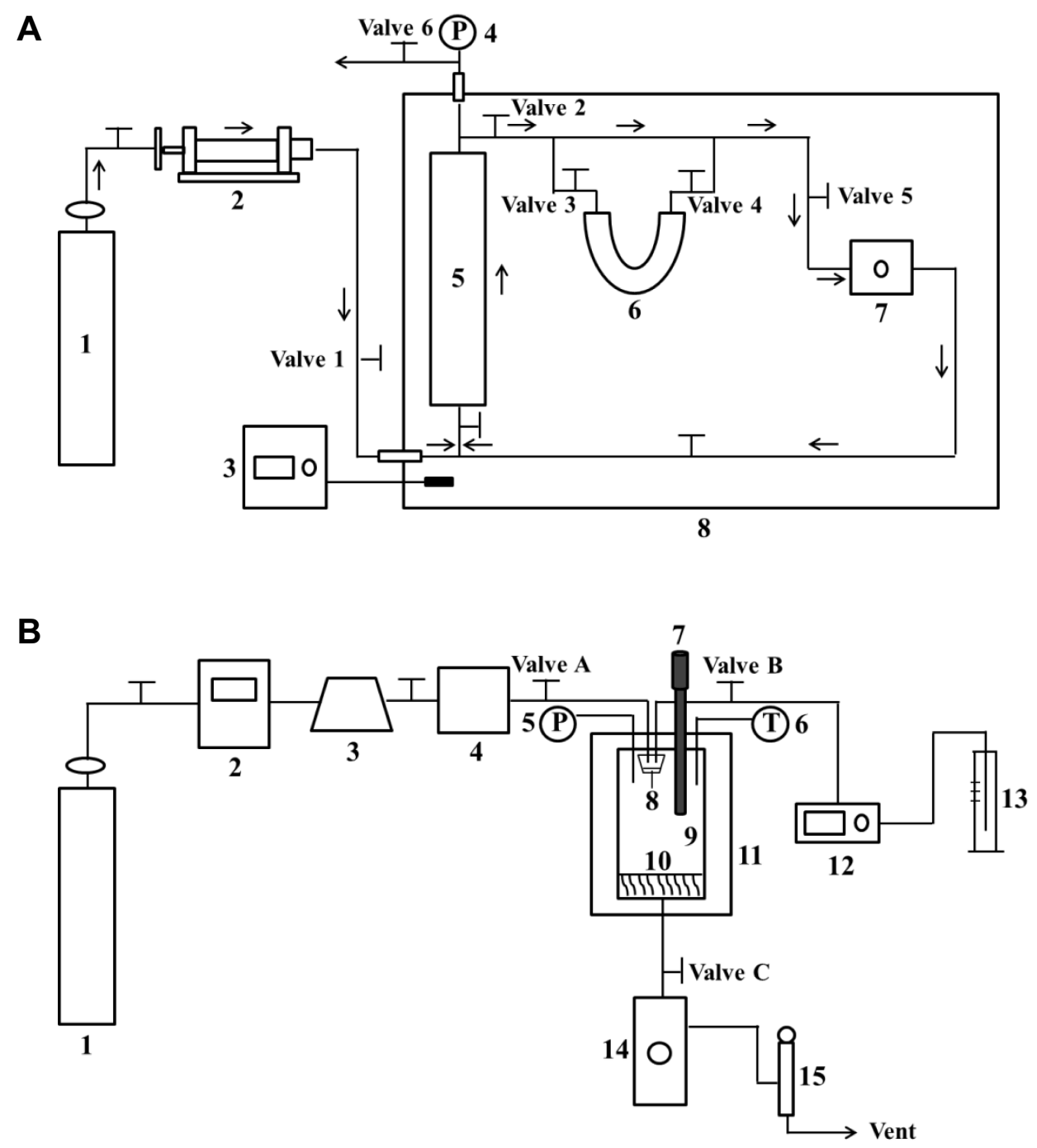

Figure I (A) Schematic diagram of the experimental apparatus for solubility measurement of breviscapine in supercritical CO2; (B) Schematic diagram of the apparatus used for ultrasound-assisted solution-enhanced dispersion by supercritical fluids.

Note: (A): I, CO2 cylinder; 2, High-pressure syringe pump; 3, Temperature sensor; 4, Pressure sensor; 5, Saturation cell; 6, U-sample collection tube; 7, Circulating pump; 8. Thermostat; (B): I, CO2 cylinder; 2, Refrigerator; 3, High-pressure pump; 4, Stabilization tank; 5, Pressure sensor; 6, Temperature sensor; 7, Ultrasonic rod; 8, Nozzle; 9 , View vessel; 10, Filter; II, Thermostats; 12, High-pressure constant flow pump; 13, Graduated flask; 14, Separator; I5, Wet gas meter.

spraying was finished, valve B was closed and fresh SCF$\mathrm{CO}_{2}$ was continuously delivered for 30 min to clean up the residual organic solvent in the products. Finally, the inlet valve was closed, while outlet remained open to relieve pressure until all gas was removed. The product in the view vessel was collected for further use.

The formulation and process parameters of BRE-MSNs were investigated by using single factor design. Then, the significant factors were optimized by central composite design (CCD) with Design Expert software (Version 8.0.6). This experimental design was used to study the effects of independent variables on various dependent variables. The three independent variables of significant factors were the pressure of view vessel $\left(\mathrm{X}_{1}\right)$, ultrasonic power $\left(\mathrm{X}_{2}\right)$, and the ratio of drug to the carrier $\left(\mathrm{X}_{3}\right)$, respectively. The dependent variables were the particle size and drug loading (DL), which were labeled as $\mathrm{Y}_{1}$ and $\mathrm{Y}_{2}$. The complete design comprised 20 experimental points to establish optimum conditions for the preparation of BRE-MSNs. The non-linear quadratic model was generated by the design as the follows:

$$
\begin{aligned}
Y= & b_{0}+b_{1} X_{1}+b_{2} X_{2}+b_{3} X_{3}+b_{12} X_{1} X_{2}+b_{13} X_{1} X_{3} \\
& +b_{23} X_{2} X_{3}+b_{11} X_{1}^{2}+b_{22} X_{2}^{2}+b_{33} X_{3}^{2}
\end{aligned}
$$

where $Y$ is the predicted response (dependent variable) associated with each factor level combination, as 
expressed in terms of the particle size and DL for the formulation variables; $b_{0}$ is an intercept, and $b_{1}$ to $b_{33}$ are the regression coefficients.

BRE-MSNs were also prepared using the SIV method with modifications to optimize the conditions. ${ }^{35}$ For DL, BRE was first dissolved in methanol in a beaker $(5 \mathrm{mg} /$ $\mathrm{mL}$ ). Then, the MSNs were added into the solution with the drug/MSNS mass ratio of $1: 3$ and the mixture was sonicated for $15 \mathrm{~min}$ at $40{ }^{\circ} \mathrm{C}$. After stirring for $24 \mathrm{~h}$, BRE-MSNs were collected by centrifugation at $10,000 \mathrm{rpm}$. Finally, the product was dried at $45{ }^{\circ} \mathrm{C}$ in vacuum for $24 \mathrm{~h}$ to remove the residual organic solvent. The product was collected and placed in a vacuum dryer for further use.

\section{Drug Loading}

BRE-MSNs were dispersed in a certain large volume of methanol. Then, BRE was completely extracted from MSNs by ultrasound and centrifugation $(10,000 \mathrm{rpm}, 10$ min). The drug content was measured with HPLC, and the DL was calculated by the following Equations 2:

$$
\mathrm{DL}(\%)=\frac{\text { Amount of BRE in solid dispersion }}{\text { Weight of solid dispersion }} \times 100
$$

\section{Particle Size Distribution and Zeta Potential Analysis}

A Nano ZS90 Zetasizer (Malvern Instruments, Malvern, UK) was used to assess the mean diameter, polydispersity index (PDI) and zeta potential of BRE-MSNs. All the samples were appropriately dispersed with deionized water. The values obtained are the average of three replicates.

\section{Characterization of BRE-MSNs}

Transmission electron microscopy (TEM) was done using a JEM-1400 TEM instrument (JEOL, Ltd., Tokyo, Japan) to determine the shape and physical appearance of samples.

The Brunauer-Emmett-Teller (BET) specific surface area, pore volume, and pore size of the prepared samples were recorded from nitrogen adsorption desorption isotherms by a TriStar II 3020 V1.02 instrument (Micromeritics Instrument Corp., Norcross, GA, USA).

A differential scanning calorimeter (DSC) (NETZSCHGeratebau GmbH, Selb, Germany) was also used to determine the physical state of the drug in the carriers. DSC scans were performed at $40-200^{\circ} \mathrm{C}$ with a heating rate of $10^{\circ} \mathrm{C} / \mathrm{min}$

X-ray diffraction (XRD) analysis of BRE-MSNs was done using a D8 Advance X-ray diffractometer (Bruker, Karlsruhe, Germany) at an operating voltage of $40 \mathrm{kV}$ and a current of $30 \mathrm{~mA}$. Samples were scanned over the range of $7-50^{\circ} 2 \theta$ at a step size of $0.02^{\circ}$ and a scan speed of $5 \% \mathrm{~min}$

\section{Solvent Residue Determination}

The solvent residue (methanol) of the products prepared by different methods was determined by gas chromatography to compare the solvent removal efficiency. An Agilent 7890A gas chromatography system coupled with a flame ionization detector (Agilent, CA, USA), and an Agilent 19091N-113 HPINNOWAX capillary column $(30 \mathrm{~m} \times 0.32 \mathrm{~mm} \times 0.25 \mathrm{~mm}$, Agilent, CA, USA) was employed for the experimental determination. The operating conditions of gas chromatography were as follows: injection temperature $=180{ }^{\circ} \mathrm{C}$; column oven temperature $=125^{\circ} \mathrm{C}$; detector temperature $=200{ }^{\circ} \mathrm{C}$; nitrogen gas flow rate $=45 \mathrm{~mL} / \mathrm{min}$; air flow rate $=300 \mathrm{~mL} / \mathrm{min}$; and hydrogen gas flow rate $=45 \mathrm{~mL} / \mathrm{min}$.

\section{Stability Study}

The accelerated stability test was conducted to assess the physical stability of the BRE-MSNs at a temperature of $313 \pm 2 \mathrm{~K}$ and a relative humidity of $75 \pm 5 \%$. Then, samples were removed and studied for the change in particle size and DL at $0,1,2,3$, and 6 months.

\section{In vitro Release Studies}

The release study of BRE from the MSNs was carried out using the small vessel method. Phosphate buffer solution ( $\mathrm{pH}$ 6.8) containing $0.3 \%$ sodium dodecyl sulfate was employed as a dissolution medium. The samples equivalent to $2 \mathrm{mg}$ of BRE were put into a conical flask with the dissolution medium maintained at $37^{\circ} \mathrm{C} \pm 0.5^{\circ} \mathrm{C}$ stirring at $100 \mathrm{rpm} .0 .5 \mathrm{~mL}$ of sample was withdrawn at a predetermined time interval and replaced with the same amount of fresh medium. All the samples were determined by HPLC and the experiments were performed in triplicates.

\section{Pharmacokinetic study}

Wistar rats were randomly divided into three groups $(\mathrm{n}=6)$ : pure BRE powder, BRE-MSNs-SIV and BRE-MSNsUSEDS. All rats were administered orally an equivalent BRE dose of $20 \mathrm{mg} / \mathrm{kg}$. Then, $0.5 \mathrm{~mL}$ blood samples were collected via the eye socket vein at predetermined time 
points and stored in heparin-coated centrifuge tubes. Blood samples were centrifuged (Eppendorf, Hamburg, Germany) at $4000 \mathrm{rpm}$ for $10 \mathrm{~min}$ at $4^{\circ} \mathrm{C}$ to separate the plasma. Before analysis, an aliquot of $0.2 \mathrm{~mL}$ plasma was vortex mixed with $1 \mathrm{~mL}$ acetonitrile for $5 \mathrm{~min}$ and then centrifuged for $15 \mathrm{~min}$ at $4000 \mathrm{rpm}$. The supernatant layer was collected and dried under nitrogen. Finally, the residue was redissolved in $100 \mu \mathrm{L}$ methanol, centrifuged at $10,000 \mathrm{rpm}$ for $10 \mathrm{~min}$ and $20 \mu \mathrm{L}$ aliquot was injected for HPLC analysis. Pharmacokinetic data were calculated by a model-independent method, using DAS 2.1.1 computer program. Pharmacokinetic parameters like peak plasma concentration $\left(\mathrm{C}_{\max }\right)$, time of peak plasma concentration $\left(\mathrm{T}_{\max }\right)$, mean retention time $\left(\mathrm{MRT}_{0-\infty}\right)$ and area under the plasma concentration-time curve $\left(\mathrm{AUC}_{0-\infty}\right)$ were calculated from the plasma concentration time profile.

\section{In vitro Cytotoxicity Assay}

We performed the 3-(4,5-dimethylthiazol-2-yl)-2,5-diphenyltetrazolium bromide (MTT)-assay on Caco-2 cells to assess the in vitro intestinal toxicity of the formulations. The cells were plated into 96 -well plates at a density of $4 \times$ $10^{3}$ cells/well, and incubated at $37^{\circ} \mathrm{C}$ under a humid atmosphere with $3.5 \% \mathrm{CO}_{2}$ before the assay. Then, the cells were treated with $20 \mu \mathrm{L}$ of different concentrations of test solutions. After incubation for $48 \mathrm{~h}, 20 \mu \mathrm{L}$ MTT was added to each well, and the cells were incubated for another $4 \mathrm{~h}$. MTT crystals were dissolved by adding $200 \mu \mathrm{L}$ of dimethylsulfoxide and the plate was stirred at room temperature for 10 min, followed by photometric determination of the absorbance at $570 \mathrm{~nm}$ using a microplate reader (Biotek Synergy HT; Gene Company Limited, Hong Kong, China). The experiments were performed in triplicate.

\section{Statistical analysis}

Results are presented as the mean \pm standard deviation. Statistical data were analyzed by multivariate linear regression for uniform design experiments, and the comparisons were made with one-way analysis of variance using IBM SPSS Statistics 21.0 software. A value of $P<0.05$ was used to determine significance.

\section{Results and Discussion}

\section{Solubility Determination of BRE}

According to the literature, ${ }^{36}$ the principle of the USEDS method is that the solvent is soluble in $\mathrm{SCF}-\mathrm{CO}_{2}$ while the solute is insoluble in $\mathrm{SCF}-\mathrm{CO}_{2}$, which leads to the rapid precipitation of the solute. Therefore, the application of the USEDS method is of great importance for the drugs with limited solubility in SCF-CO $\mathrm{CO}_{2}$. In order to determine the feasibility of preparing BRE-MSNs with the USEDS method, we initially measured the solubility of BRE in $\mathrm{SCF}-\mathrm{CO}_{2}$. Figure 2 shows the effect of pre-saturation time on the solubility of BRE in SCF-CO $\mathrm{C}_{2}$. The solubility of BRE increased significantly within $75 \mathrm{~min}$ and had little change after $75 \mathrm{~min}$. Considering the experimental efficiency, we chose the pre-saturation time of $75 \mathrm{~min}$. Under the conditions of constant temperature, the solubility increased with the increase in pressure. This could be explained by the fact that the increase in pressure led to the increase in solvent density and enhanced the specific interaction between the dissolved substances and the solvent molecules. Increasing the density of $\mathrm{CO}_{2}$ also increases the polarity of its molecules. ${ }^{37}$ As the temperature increased, the density of SCF- $\mathrm{CO}_{2}$ decreased, leading to a decrease in solvent capacity. Thus, the solubility of BRE decreased. It should be noted that the solubility of $\mathrm{BRE}$ in $\mathrm{SCF}-\mathrm{CO}_{2}$ was extremely low $(<2 \mathrm{mg} / \mathrm{mol})$ in all cases, indicating the feasibility of applying the USEDS method to the preparation of BRE-MSNs.

\section{Technology and Formulation Optimization Study}

Before CCD, we investigated the influence factors including pressure, temperature, the flow rate of the solution, ultrasound power and ratio of drug to the carrier, with the particle size and DL as the evaluation indexes. Finally, we selected three factors (pressure, ultrasound power and the ratio of drug to carrier) that significantly affected the particle size and DL for further optimization. In order to evaluate the influence of various factors on the preparation process of USEDS, 17 experiments were performed and are listed in Table 1. As shown in Table 1, the values of $Y_{1}$ and $Y_{2}$ responses were in the range of 160.9 to $223.3 \mathrm{~nm}$ and 4.91 to $9.06 \%$, respectively. The regression coefficients of quadratic polynomial equations were calculated with experimental data, and the significance of each regression coefficient was statistically evaluated by ANOVA. The independent variable correlation coefficient with significant t-values $(P \leq 0.05)$ was adopted to establish the model. The lower p-value indicates the higher significant influence of the independent variable on the response. 

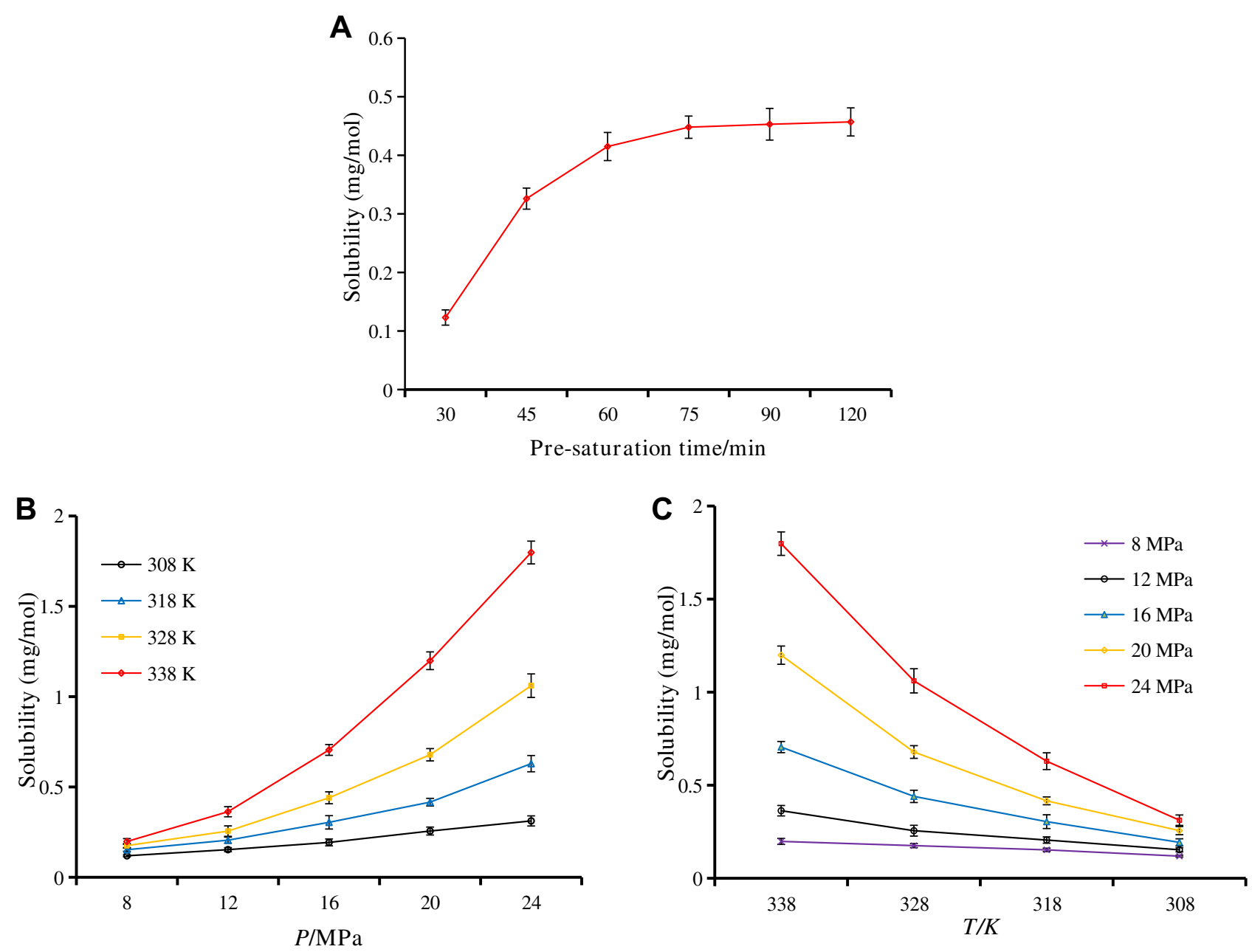

Figure 2 Solubility of breviscapine in supercritical $\mathrm{CO}_{2}$ under various conditions. (A) Pre-saturation time; (B) pressure; and (C) temperature.

The data obtained from CCD experiments were fitted to a second-order polynomial model and the final equations were represented by the following equation:

$$
\begin{aligned}
Y_{1}= & 171.14+0.45 X_{1}-13.03 X_{2}+14.75 X_{3} \\
& +3.5 X_{1} X_{2}-0.9 X_{1} X_{3}-6.5 X_{2} X_{3}+15.05 X_{1}{ }^{2} \\
& +16.05 X_{2}^{2}-3.69 X_{3}^{2} \\
Y_{2}= & 8.19+0.036 X_{1}+0.24 X_{2}+1.53 X_{3}+0.21 X_{1} X_{2} \\
& -0.17 X_{1} X_{3}-0.13 X_{2} X_{3}-0.30 X_{1}^{2}+-0.32 X_{2}{ }^{2} \\
& -0.73 X_{3}^{2}
\end{aligned}
$$

The predicted R-squared values of $\mathrm{Y}_{1}$ and $\mathrm{Y}_{2}$ were 0.821 and 0.858 for particle size and DL, respectively, indicating the adequacy of the model to predict the response of particle size and DL. The optimized preparation with small particle size and high DL was obtained with a pressure of $14.45 \mathrm{MPa}$, ultrasound power of 74.34
W and value of drug: MSNs at the ratio of 0.23 , respectively. The comparison of predicted and experimental data of the dependent variable is listed in Table 2. These data showed that the experimental data approached the predicted data with low percentage bias, suggesting that the optimized formulation was reasonable and reliable.

Contours and three-dimensional surface plots for the most statistically significant variables on the evaluated parameters are shown in Figure 3. As can be seen in Figure 3A, in association with Equation 3, the particle size was significantly influenced by ultrasound power $\left(\mathrm{X}_{2}\right)$ and the ratio of drug to the carrier $\left(\mathrm{X}_{3}\right)$ with large coefficients $(-13.03$ and 14.75), The results suggested that particle size decreased with the increase ultrasonic power, which may be attributed to better dispersion of particles with an increase in ultrasonic power. In addition, the particle size increased with an increase in drug concentration. This might be due to the solute concentration of the system competing against volume 
Table I Coded Levels and Measured Responses for the 17 Experiment Formulation Runs

\begin{tabular}{|l|l|l|l|l|l|}
\hline NO & $\begin{array}{l}\mathbf{X}_{\mathbf{1}} \\
(\mathbf{M P a})\end{array}$ & $\begin{array}{l}\mathbf{X}_{\mathbf{2}} \\
\mathbf{( W )}\end{array}$ & $\mathbf{X}_{\mathbf{3}}$ & $\begin{array}{l}\text { Size (nm) } \\
\left(\mathbf{Y}_{\mathbf{1}}\right)\end{array}$ & $\begin{array}{l}\mathbf{D L}(\%) \\
\left(\mathbf{Y}_{\mathbf{2}}\right)\end{array}$ \\
\hline 1 & 13 & 30 & 0.19 & 223.3 & 7.54 \\
2 & 19 & 30 & 0.19 & 207.1 & 6.75 \\
3 & 13 & 90 & 0.19 & 190.4 & 7.97 \\
4 & 19 & 90 & 0.19 & 188.2 & 8.03 \\
5 & 13 & 60 & 0.13 & 160.9 & 4.91 \\
6 & 19 & 60 & 0.13 & 173.7 & 5.76 \\
7 & 13 & 60 & 0.25 & 193.1 & 8.89 \\
8 & 19 & 60 & 0.25 & 202.3 & 9.06 \\
9 & 16 & 30 & 0.13 & 175.8 & 5.72 \\
10 & 16 & 90 & 0.13 & 162.6 & 6.07 \\
11 & 16 & 30 & 0.25 & 217.4 & 8.46 \\
12 & 16 & 90 & 0.25 & 178.2 & 8.31 \\
13 & 16 & 60 & 0.19 & 170.6 & 8.26 \\
14 & 16 & 60 & 0.19 & 169.3 & 8.12 \\
15 & 16 & 60 & 0.19 & 167.3 & 8.37 \\
16 & 16 & 60 & 0.19 & 174.4 & 8.04 \\
17 & 16 & 60 & 0.19 & 174.1 & 8.15 \\
\hline
\end{tabular}

Notes: $X_{1}$, Pressure; $X_{2}$, Ultrasound power; $X_{3}$, value of drug: carrier. Abbreviation: DL, drug loading.

Table 2 Comparison of the Experimental and Predicted Values of BRE-MSNs-USEDS Under Predicted Optimum Conditions

\begin{tabular}{|l|l|l|l|}
\hline $\begin{array}{l}\text { Measured } \\
\text { Responses }\end{array}$ & $\begin{array}{l}\text { Predicted } \\
\text { Values }\end{array}$ & $\begin{array}{l}\text { Experimental } \\
\text { Values }\end{array}$ & $\begin{array}{l}\text { Bias } \\
\text { (\%) }\end{array}$ \\
\hline YI(Size) & $174.32 \mathrm{~nm}$ & $177.24 \mathrm{~nm}$ & -1.69 \\
Y2(DL) & $8.86 \%$ & $8.63 \%$ & 2.59 \\
\hline
\end{tabular}

Note: Bias was calculated as (predicted value - observed value)/predicted value $\times$ $100 \%$.

expansion. As the solute concentration increased, the nucleation obtained at the lower volume expansion was diminished. Therefore, the nucleus would grow for a longer time to form a larger particle size. ${ }^{38}$

The results in $\mathrm{Y}_{2}$ Equation 4 and Figure 3B show that the ratio of drug to MSNs $\left(\mathrm{X}_{3}\right)$ had a highly positive effect on the DL of MSNs indicating that drug concentration was the determining factor for drug loading. It was obvious that an increase in drug concentration resulted in an increase in DL, which could be attributed to the fact that more drug could be loaded into MSNs with increasing drug concentration.

\section{Comparison of BRE-MSNs Prepared by Different Methods}

The particle size, PDI, zeta potential and DL of BREMSNs prepared by the SIV method and the USEDS method are shown in Table 3. Compared with the SIV method, the USEDS method could form smaller particles with higher DL. The mean particle size and DL of BREMSNs-USEDS were $177.24 \mathrm{~nm}$ and 8.63\%, while BREMSNs-SIV were $221.31 \mathrm{~nm}$ and $5.12 \%$, respectively. In addition, BRE-MSNs-USEDS have a smaller PDI and a higher zeta potential, indicating that they have better stability. The USEDS method utilized the characteristics of SCF and the special preparation process to obtain BREMSNs with better performance. Therefore, the USEDS method has certain advantages in preparing drug-loaded MSNs over the conventional SIV methods.

\section{Transmission Electron Microscope}

TEM images revealed major features about the particle size, surface morphology and inner structure of BRE-MSNs. As shown in Figure 4, most particles were uniform and roughly spherical in shape with average particle size. Furthermore, a well-ordered pore structure with a linear array of pores arranged at regular intervals was presented in the images, which was similar to the structure observed by Li et al. ${ }^{39}$ The BRE-MSNs prepared by the USEDS method were smaller and more dispersed than those prepared by the SIV method, which corroborated the particle size measurements. In addition, the particle size distribution shown in the TEM images is smaller than that measured by dynamic light scattering (DLS) method. It may be because with the DLS method was difficult to distinguish clustered particles, resulting in exaggerated average particle size of MSNs. Similar conclusions were reported by Jia et al. ${ }^{40}$

\section{Nitrogen Adsorption-Desorption Measurements}

For bare MSNs, the adsorption-desorption isotherm was a typical type IV sorption isotherm. As shown in Table 4 and Figure 5, the BET surface, total pore volume, and BJH average pore diameter of BRE-MSNs-SIV and BRE-MSNs -USEDS were lower than those of the bare MSNs. These results suggested that BRE was loaded into the pores of MSNs. Moreover, the BET surface, total pore volume, and average pore diameter of BRE-MSNs-USEDS were lower than those of BRE-MSNs-SIV, indicating the DL of BREMSNs-USEDS was higher than that of BRE-MSNs-SIV.

Differential scanning calorimetry (DSC) and X-ray diffraction (XRD)

DSC thermograms of the pure drug, MSNs, physical mixtures, BRE-MSNs-SIV and BRE-MSNs-USEDS are 
A

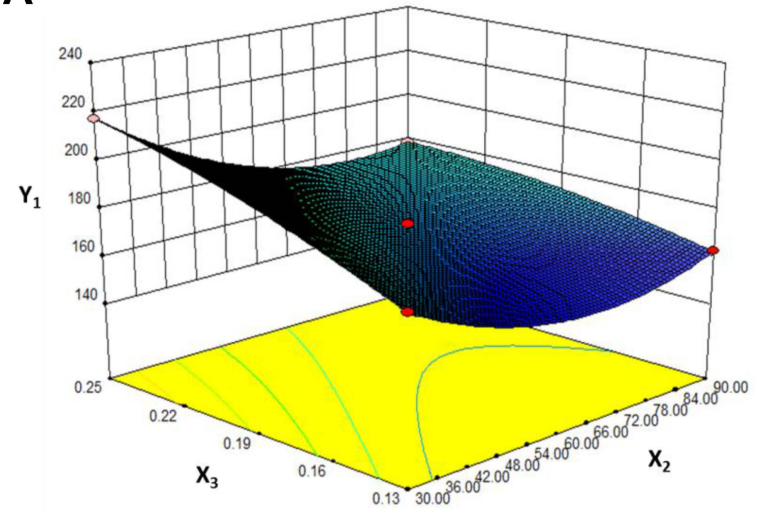

B

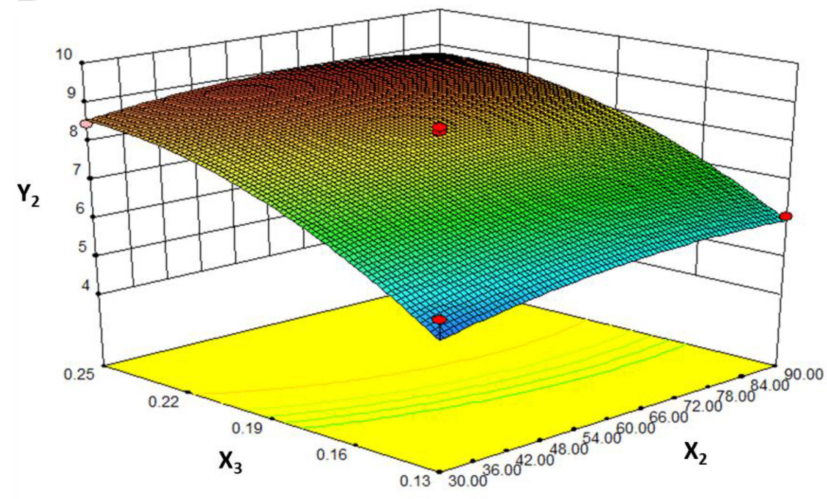

Figure 3 Response surface models showing the influence of the factors on the responses.

Notes: Three-dimensional response surface plots showing the effects of $\left(\mathrm{X}_{2}\right)$ ultrasound power and $\left(\mathrm{X}_{3}\right)$ value of drug: carrier on the responses of $\mathrm{YI}(\mathbf{A})$ and $\mathrm{Y} 2(\mathbf{B})$. $\mathrm{YI}$ is the response of the mean particle size. $Y 3$ is the response of drug loading.

presented in Figure 6A. The endothermic peak of pure BRE was at $134.1^{\circ} \mathrm{C}$. The DSC curve of PMs exhibited the same endothermic peak with pure BRE. However, MSNs, BRE-MSNs-SIV and BRE-MSNs-USEDS showed no endothermic peak of BRE in their DSC curves, which indicated that BRE loaded into MSNs had transferred to a non-crystalline or amorphous form.

Figure 6B displays the XRD diffractograms of the pure drug, MSNs, physical mixtures, BRE-MSNs-SIV and BRE-MSNs-USEDS. The pure drug and physical mixtures exhibited characteristic crystalline peaks at $2 \theta$ of 8.0, 10.1, 14.3, 16.1, 25.8 and 26.8 ${ }^{\circ}$. However, MSNs did not show any obvious diffraction peaks due to their amorphous nature. $^{41}$ For BRE-MSNs-SIV and BRE-MSNs-USEDS, the diffraction peaks of BRE disappeared, which indicated that BRE loaded into MSNs was in a non-crystalline or amorphous form. The results of XRD were in complete

Table 3 The Particle Size, PDI, Zeta Potential and DL of the BRE-MSNs-SIV and BRE-MSNs-USEDS $(n=3)$

\begin{tabular}{|l|l|l|l|l|}
\hline Sample & $\begin{array}{l}\text { Particle } \\
\text { Size }(\mathbf{n m})\end{array}$ & PDI & $\begin{array}{l}\text { Zeta } \\
\text { Potential } \\
(\mathbf{m V})\end{array}$ & $\begin{array}{l}\text { DL } \\
\mathbf{( \% )}\end{array}$ \\
\hline $\begin{array}{l}\text { BRE-MSNs- } \\
\text { SIV }\end{array}$ & $221.31 \pm 7.18$ & $\begin{array}{l}0.154 \\
\pm 0.012\end{array}$ & $-25.9 \pm 2.6$ & $\begin{array}{l}5.12 \\
\pm 0.35\end{array}$ \\
\hline BRE-MSNs- \\
USEDS & $177.24 \pm 5.72 *$ & $\begin{array}{l}0.269 \\
\pm 0.016 *\end{array}$ & $-27.3 \pm 2.9$ & $\begin{array}{l}8.63 \\
\pm 0.44^{*}\end{array}$ \\
\hline
\end{tabular}

Note: *p $\leq 0.05$ compared with the BRE-MSNs-SIV.

Abbreviations: PDI, polydispersity index; DL, drug loading; BRE-MSNs-SIV, breviscapine loaded mesoporous silica nanoparticles prepared by the solution impregnation-evaporation method; BRE-MSNs-USEDS, breviscapine loaded mesoporous silica nanoparticles prepared by the ultrasound-assisted solution-enhanced dispersion by supercritical fluids. agreement with those of DSC. The amorphous state of BRE within MSNs might contribute to its increased drug solubility. ${ }^{42}$

\section{Residual Solvent}

It has been reported that solvent residues present in the preparation may affect its stability and safety. ${ }^{43}$ Therefore, we evaluated the solvent residual quantity of formulations prepared by different methods. Methanol residue was determined in the BRE-MSNs prepared in this study, and the results are shown in Figure 7. In the pharmaceutical industry, the presence of a certain amount of methanol that does not exceed the limit ( $3000 \mathrm{ppm})$ is considered acceptable. The USEDS method removes the solvent from the preparation depending on the principle of solvent dissolution in SCF- $\mathrm{CO}_{2}$. In BRE-MSNs-USEDS, residual methanol was reduced to $152 \mathrm{ppm}$ after being dried for only 30 min, while in BRE-MSNs-SIV, the residual solvent remained at $317 \mathrm{ppm}$ after $24 \mathrm{~h}$ of drying. This indicates that the solvent removal efficiency of the USEDS method was much higher than that of the SIV method.

\section{Stability Test}

Stability is one of the important indexes to evaluate the properties of the preparation. The results of accelerated stability testing are given in Figure 8. From the obtained results, the particle sizes of BRE-MSNs-USEDS and BREMSNs-SIV increased from $177.24 \mathrm{~nm}$ to $186.32 \mathrm{~nm}$ and $221.31 \mathrm{~nm}$ to $269.64 \mathrm{~nm}$ within 6 months, respectively. In addition, the DL of BRE-MSNs-USEDS and BRE-MSNsSIV decreased from $8.63 \% \mathrm{~nm}$ to $8.19 \%$ and $5.12 \%$ to $4.13 \%$, respectively. It indicated that the change in particle 

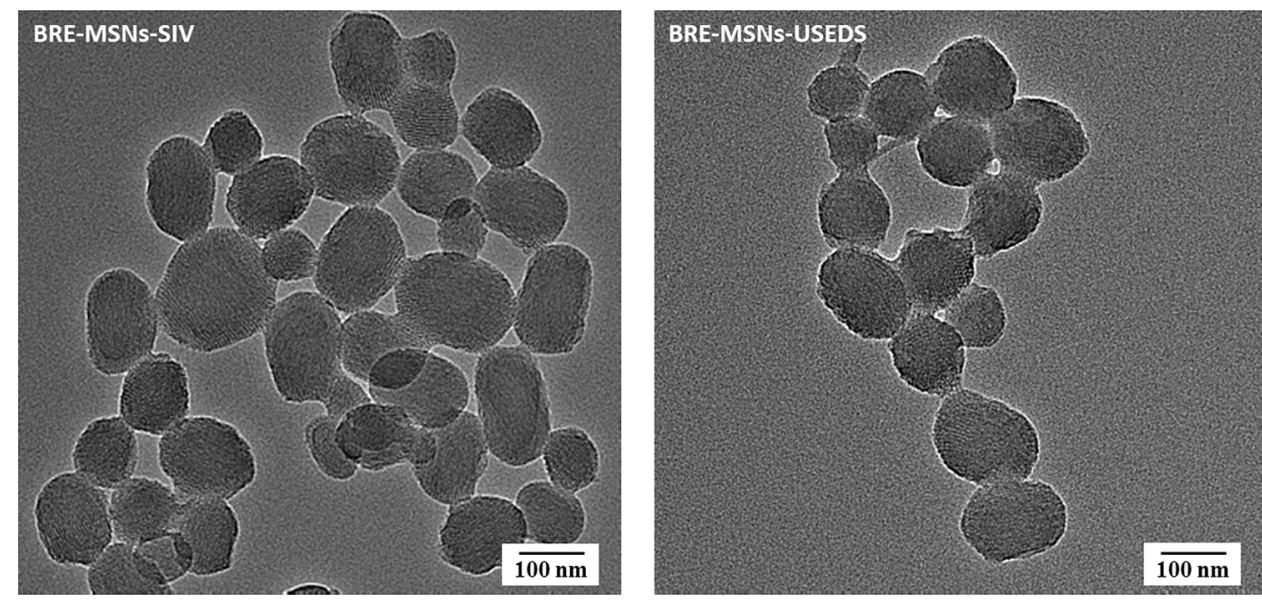

Figure 4 Transmission electron micrograph of BRE-MSNs-SIV and BRE-MSNs-USEDS.

Abbreviations: BRE-MSNs-SIV, breviscapine-loaded mesoporous silica nanoparticles prepared by the solution impregnation-evaporation method; BRE-MSNs-USEDS, breviscapine-loaded mesoporous silica nanoparticles prepared by the ultrasound-assisted solution-enhanced dispersion by supercritical fluids.

size and DL of BRE-MSNs-USEDS was smaller than that of BRE-MSNs-SIV within 6 months. This might because the less residual solvent reduced the aggregation of the particles. In addition, the drug was better loaded into the channels of MSNs by using the USEDS method so that fewer drugs would remain on the surface of MSNs. These two advantages determined that BRE-MSNs-USEDS had a better stability.

\section{In vitro Dissolution Studies}

The dissolution profiles of the samples are shown in Figure 9. The BRE-MSNs showed a more rapid dissolution of BRE than the BRE powder. During the first $30 \mathrm{~min}$, the solubility of BRE powder was about $26 \%$, while the solubility of BREMSNs was more than $80 \%$. BRE-MSNs showed a sudden release phenomenon at the first stage, which was speculated to be the precipitation and adsorption of drugs on the surface of MSNs. Finally, the solubility of BRE-MSNs was significantly higher than that of BRE powder when the solution balance

Table 4 Material Properties of MSNs Analyzed by Nitrogen Adsorption-Desorption

\begin{tabular}{|l|l|l|l|}
\hline Sample & $\begin{array}{l}\text { BET Surface } \\
\text { Area }\left(\mathbf{m}^{\mathbf{2}} / \mathbf{g}\right)\end{array}$ & $\begin{array}{l}\text { Pore } \\
\text { Volume } \\
\left(\mathbf{m}^{3} / \mathbf{g}\right)\end{array}$ & $\begin{array}{l}\text { Pore } \\
\text { Size } \\
(\mathbf{n m})\end{array}$ \\
\hline Blank-MSNs & 1029.2 & 1.1 & 4.1 \\
BRE-MSNs-SIV & 511.5 & 0.4 & 2.7 \\
BRE-MSNs-USEDS & 456.3 & 0.3 & 2.5 \\
\hline
\end{tabular}

Abbreviations: BET, Brunauer-Emmett-Teller; MSNs, mesoporous silica nanoparticles; BRE-MSNs-SIV, breviscapine loaded mesoporous silica nanoparticles prepared by the solution impregnation-evaporation method; BRE-MSNs-USEDS, breviscapine loaded mesoporous silica nanoparticles prepared by the ultrasoundassisted solution-enhanced dispersion by supercritical fluids. was reached. This might be due to the fact that polycrystalline had relatively less effect on the dissolution than the formation of a high energy amorphous phase. ${ }^{44}$ On the other hand, according to the Ostwald-Freundlich equation, the decrease in particle size led to the increase in specific surface area, so the saturation solubility also increased. ${ }^{45}$ However, BREMSNs-USEDS had a larger cumulative release than BREMSNs-SIV owing to their smaller particle size. Moreover, compared with BRE-MSNs-SIV, the burst release behavior of BRE-MSNs-USDES is smaller, which depends on its better adsorption effect on the drug, resulting in less drug residues on the surface. Thus, the drug can be released more smoothly, which can reduce its sudden release after oral absorption into blood. According to the dissolution results, it can be concluded that the increase in dissolution might lead to better oral absorption.

\section{Pharmacokinetics Study}

Generally, the bioavailability of BRE, a class II drug according to the Biopharmaceutic Classification System (BCS), is limited by its poor dissolution, and an increase in dissolution rate would result in an improvement in its bioavailability. ${ }^{46}$ By loading into MSNs, the dissolution rate of BRE significantly increased, so it could be expected that the drug bioavailability in rats would be significantly improved.

The in vivo study was adopted to investigate the pharmacokinetic behavior of BRE after the oral administration of different samples. The plasma concentration-time profiles of three BRE formulations are shown in Figure 10 and the pharmacokinetic parameters calculated from the time profiles are presented in Table 5. It is apparent in 


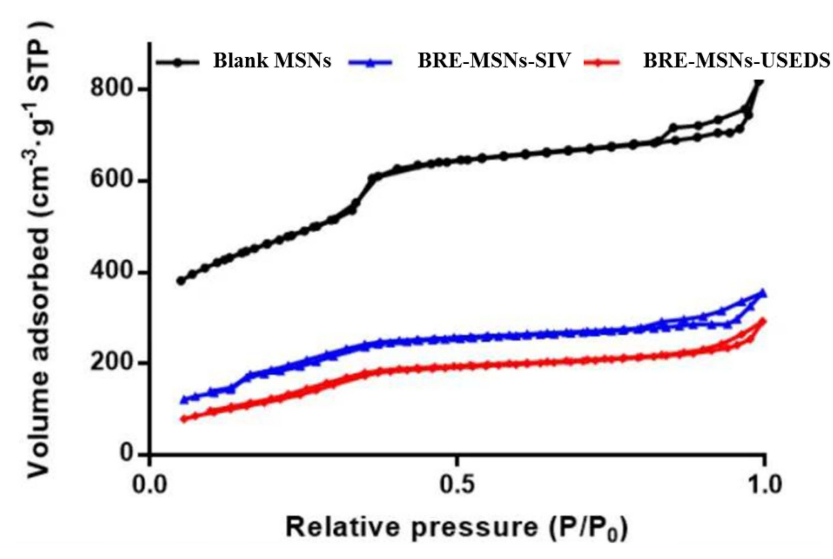

Figure $\mathbf{5}$ The nitrogen adsorption-desorption isotherms of the blank MSNs, BREMSNs-SIV and BRE-MSNs-USEDS.

Abbreviations: MSNs, mesoporous silica nanoparticles; BRE-MSNs-SIV, breviscapine-loaded mesoporous silica nanoparticles prepared by the solution impregnation-evaporation method; BRE-MSNs-USEDS, breviscapine-loaded mesoporous silica nanoparticles prepared by the ultrasound-assisted solution-enhanced dispersion by supercritical fluids.

Figure 10 that the plasma concentration profile of BREMSNs represented a greater improvement in drug absorption than the BRE powder. For the BRE powder, the $\mathrm{AUC}_{0-\infty}, \mathrm{C}_{\max }$, and $\mathrm{T}_{\max }$ were $3.07 \mu \mathrm{g} / \mathrm{h} \cdot \mathrm{mL}, 0.46 \mu \mathrm{g} /$ $\mathrm{mL}$ and $1.17 \mathrm{~h}$ after oral administration, respectively. The BRE-MSNs had significantly higher $\mathrm{AUC}_{0-\infty}$ and $\mathrm{C}_{\max }$ than $\mathrm{BRE}$ powder $(P<0.05)$. In particular, the $\mathrm{AUC}_{0-\infty}$ of BRE-MSNs-SIV and BRE-MSNs-USEDS was $\sim 1.70$-fold and 1.96-fold higher than that of BRE powder, while the $\mathrm{C}_{\max }$ were 2.17- and 2.13-fold, respectively. Thus, the increase in the oral bioavailability of BRE in solid dispersion might be attributed to the significant increase in the absorption rate of BRE due to the increased dissolution of the drug in the solid dispersion in rats. Furthermore, the differences in bioavailability between BRE-MSNs prepared by USEDS and SIV method might be attributed to the differences in the surface area of dispersed drug particles. The results suggested that the preparation of BRE-MSNs using the USEDS method might be a promising approach for improving the bioavailability of BRE.

\section{In vitro Cytotoxicity}

Since the main research objective was the preparation of oral formulation, it was necessary to investigate the toxicity of the drug and the carrier to the intestinal cells. The morphology and function of Caco-2 cells are similar to that of small intestinal epithelial cells. Therefore we selected Caco-2 cells for cytotoxicity investigation. The percentage of cell viability at different concentration ranges after $24 \mathrm{~h}$ incubation is shown in Figure 11. The viability of Caco-2 cells decreased with an increase in the concentrations of the blank MSNs, BRE powder, BREMSNs-SIV and BRE-MSNs-USEDS. Blank MSNs had no significant toxicity in the cells, as the cell viability was greater than $90 \%$. Other samples also had low cytotoxicity,

B
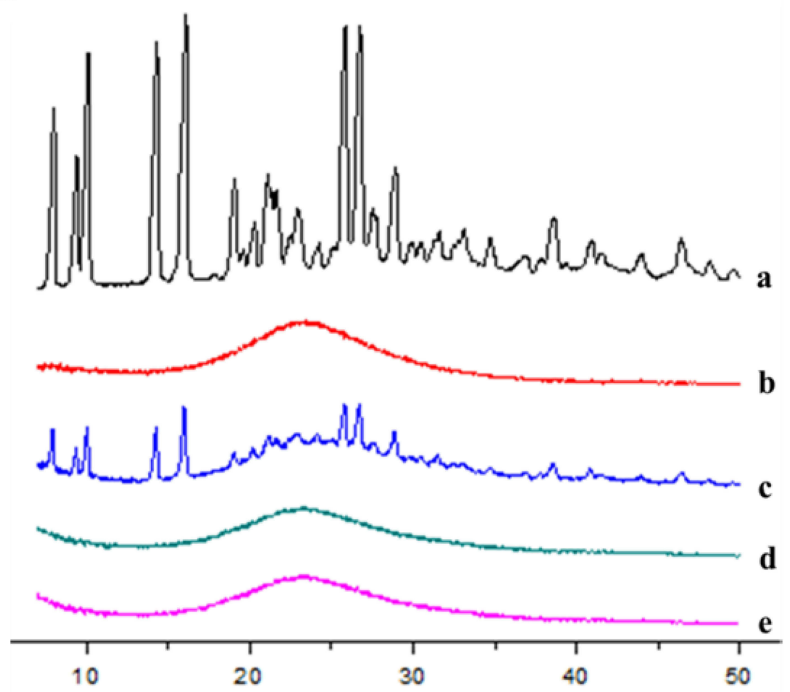

Figure 6 (A) Differential scanning calorimetry analyses and (B) X-ray diffraction analyses of (a) BRE, (b) MSNs, (c) physical mixtures, (d) BRE-MSNs-SIV and (e) BRE-MSNsUSEDS.

Abbreviations: BRE, breviscapine; MSNs, mesoporous silica nanoparticles; BRE-MSNs-SIV, breviscapine-loaded mesoporous silica nanoparticles prepared by the solution impregnation-evaporation method; BRE-MSNs-USEDS, breviscapine-loaded mesoporous silica nanoparticles prepared by the ultrasound-assisted solution-enhanced dispersion by supercritical fluids. 


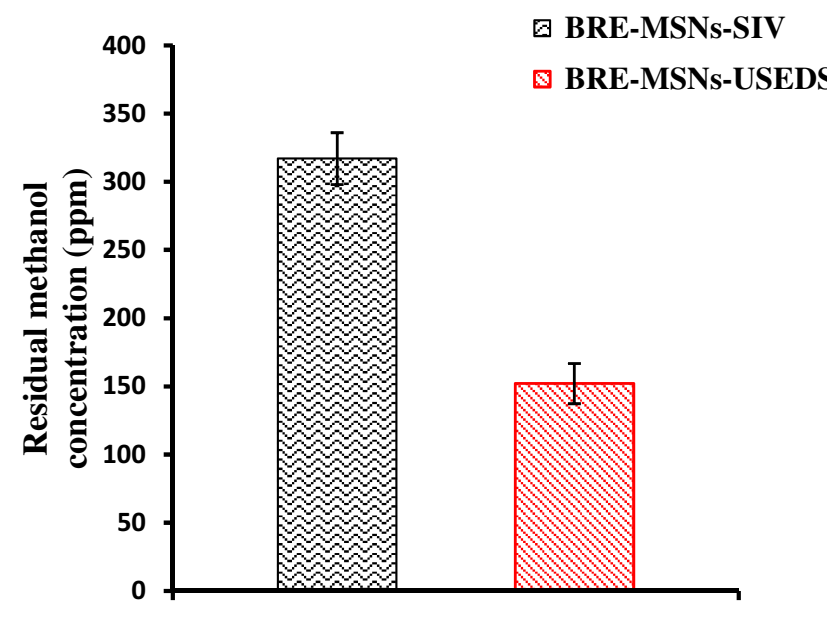

Figure 7 The concentration of residual methanol in BRE-MSNs prepared by different methods.

Abbreviations: BRE-MSNs, breviscapine-loaded mesoporous silica nanoparticles; BRE-MSNs-SIV, breviscapine-loaded mesoporous silica nanoparticles prepared by the solution impregnation-evaporation method; BRE-MSNs-USEDS, breviscapineloaded mesoporous silica nanoparticles prepared by the ultrasound-assisted solution-enhanced dispersion by supercritical fluids.

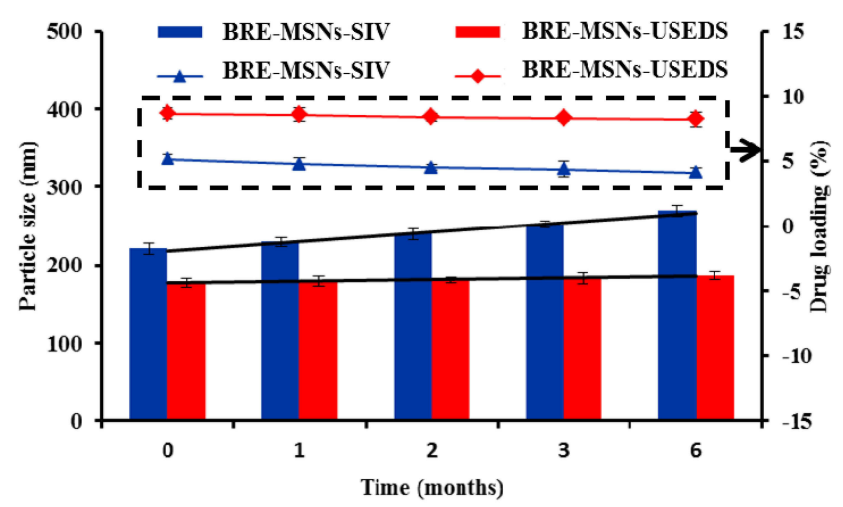

Figure 8 The accelerated stability studies for BRE-MSNs prepared by different methods.

Abbreviations: BRE-MSNs, breviscapine-loaded mesoporous silica nanoparticles; BRE-MSNs-SIV, breviscapine-loaded mesoporous silica nanoparticles prepared by the solution impregnation-evaporation method; BRE-MSNs-USEDS, breviscapineloaded mesoporous silica nanoparticles prepared by the ultrasound-assisted solution-enhanced dispersion by supercritical fluids.

with cell survival rates greater than $80 \%$. In addition, the cell survival rate of BRE-MSNs-USEDS was higher than that of BRE-MSNs-SIV, possibly due to its lower levels of residual organic solvents. These results indicated that BRE-MSNs-USEDS was biocompatible with Caco-2 cells and was well tolerated in the gastrointestinal tract.

\section{Conclusion}

In the present study, the BRE solid dispersion with MSNs as carriers were prepared using USEDS under certain conditions which were evaluated by different factors.

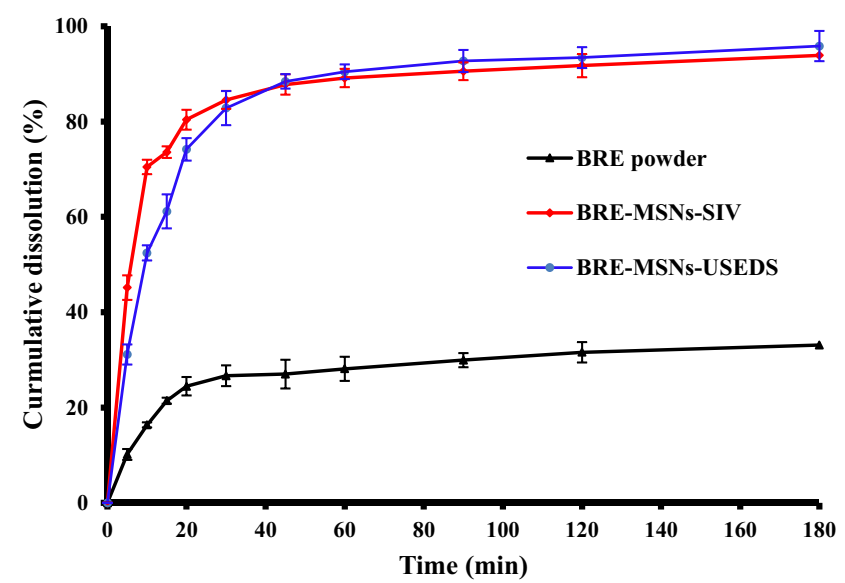

Figure 9 In vitro release of BRE,BRE-MSNs-SIV and BRE-MSNs-USEDS in phosphate buffered solution ( $\mathrm{pH}=6.8$ ).

Abbreviations: BRE, breviscapine; BRE-MSNs-SIV, breviscapine-loaded mesoporous silica nanoparticles prepared by the solution impregnation-evaporation method; BRE-MSNs-USEDS, breviscapine-loaded mesoporous silica nanoparticles prepared by the ultrasound-assisted solution-enhanced dispersion by supercritical fluids.

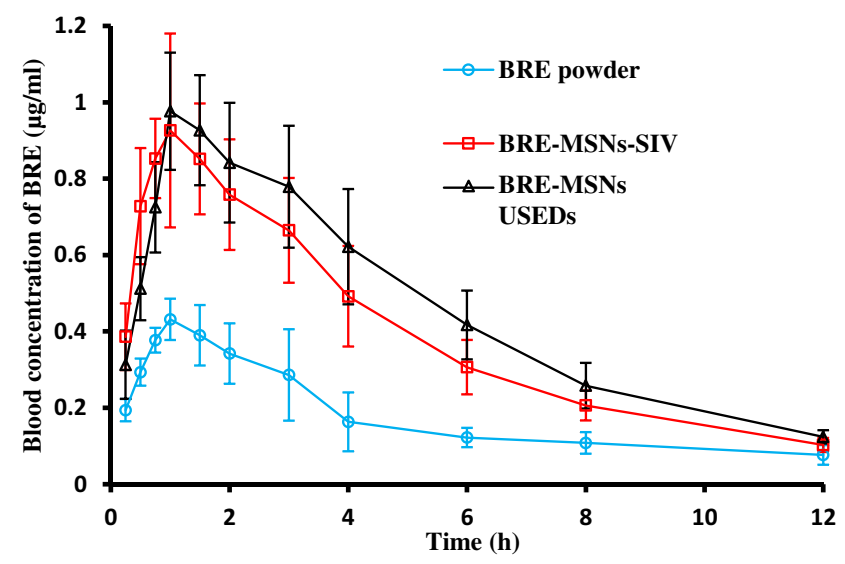

Figure 10 In vivo plasma concentration-time profiles. Levels of BRE following oral administration of BRE powder suspension, BRE-MSNs-SIV and BRE-MSNs-USEDS $(n=6)$.

Abbreviations: BRE, breviscapine; BRE-MSNs-SIV, breviscapine-loaded mesoporous silica nanoparticles prepared by the solution impregnation-evaporation method; BRE-MSNs-USEDS, breviscapine-loaded mesoporous silica nanoparticles prepared by the ultrasound-assisted solution-enhanced dispersion by supercritical fluids.

The product exhibited improved characteristics, such as larger surface, smaller particle size, higher DL, less solvent residues and better stability, as compared to the BRE-MSNs prepared by the SIV method. The dissolution rate of BRE-MSNs-USEDS was significantly higher than that of BRE powder, which could be related to the amorphous state of BRE as revealed by XRD and DSC analyses. The in vivo study indicated the enhanced effects on the oral absorption of the BRE loaded into MSNs and prepared by USEDS. Compared with other breviscapine drug delivery systems, MSN has certain advantages, such 
Table 5 Main Pharmacokinetic Parameters of the BRE Powder, BRE-MSNs-SIV and BRE-MSNs-USEDS After Oral Administration to Rats $(n=6)$

\begin{tabular}{|l|l|l|l|}
\hline $\begin{array}{l}\text { Pharmacokinetic } \\
\text { Parameters }\end{array}$ & $\begin{array}{l}\text { BRE } \\
\text { Powder }\end{array}$ & $\begin{array}{l}\text { BRE-MSNs- } \\
\text { SIV }\end{array}$ & $\begin{array}{l}\text { BRE-MSNs- } \\
\text { USEDS }\end{array}$ \\
\hline $\mathrm{C}_{\max }(\mu \mathrm{g} / \mathrm{mL})$ & $0.46 \pm 0.03$ & $1.00 \pm 0.20^{*}$ & $0.98 \pm 0.15^{*}$ \\
$\mathrm{~T}_{\max }(\mathrm{h})$ & $1.17 \pm 0.26$ & $0.96 \pm 0.29$ & $1.17 \pm 0.41$ \\
$\mathrm{MRT}_{0-\infty}(\mathrm{h})$ & $7.65 \pm 1.79$ & $5.13 \pm 0.45^{*}$ & $5.51 \pm 0.49 *$ \\
$\mathrm{AUC}_{0-\infty}(\mu \mathrm{g} / \mathrm{h} \mathrm{mL})$ & $3.07 \pm 1.17$ & $5.21 \pm 0.81^{*}$ & $6.02 \pm 1.01 *$ \\
\hline
\end{tabular}

Note: ${ }^{*} \mathrm{p} \leq 0.05$ compared with the pure BRE powder.

Abbreviations: BRE, breviscapine; MSNs, mesoporous silica nanoparticles; BREMSNs-SIV, breviscapine loaded mesoporous silica nanoparticles prepared by the solution impregnation-evaporation method; BRE-MSNs-USEDS, breviscapine loaded mesoporous silica nanoparticles prepared by the ultrasound-assisted solutionenhanced dispersion by supercritical fluids; $\mathrm{C}_{\max }$, peak plasma concentration; $\mathrm{T}_{\max }$, time peak plasma concentration; $\mathrm{MRT}_{0-\infty}$, mean retention time; $A U C_{0-\infty}$, area under the plasma concentration-time curve.

as smaller particle size than lipid emulsions, ${ }^{47}$ greater drug loading than SLN, ${ }^{2}$ and higher in vitro dissolution than liposomes. ${ }^{48}$ In addition, MSN has better stability as an inorganic material. Therefore, MSN as a carrier to improve drug solubility and oral absorption has a great application prospect. Moreover, as the preparation method of this study, the USEDS method exhibited great potential for the preparation of drug-loaded solid dispersions.

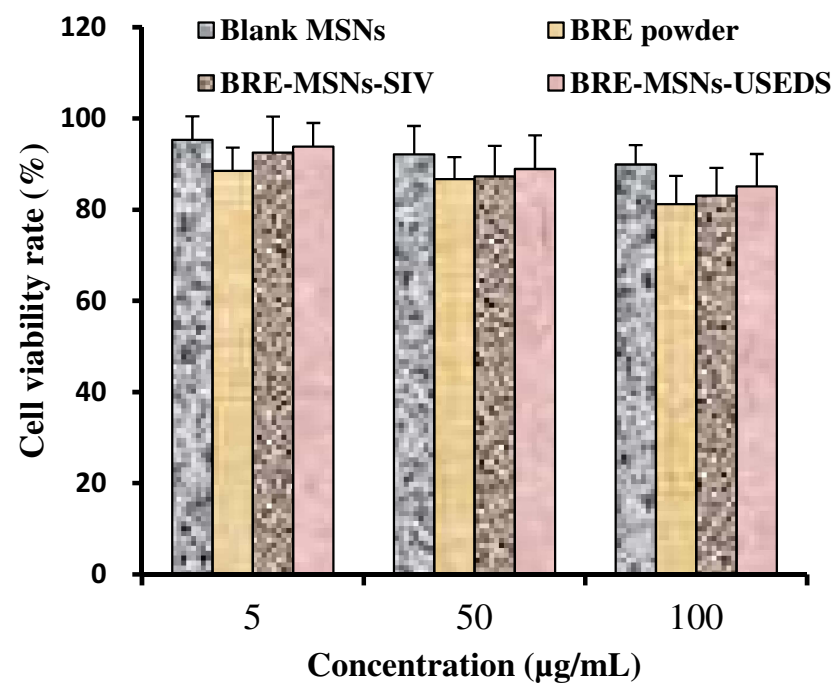

Figure I I Results of the MTT assay with Caco-2 cells. Data are given as mean \pm SD $(n=6)$.

Abbreviations: BRE, breviscapine; MSNs, mesoporous silica nanoparticles; BREMSNs-SIV, breviscapine loaded mesoporous silica nanoparticles prepared by the solution impregnation-evaporation method; BRE-MSNs-USEDS, breviscapine loaded mesoporous silica nanoparticles prepared by the ultrasound-assisted solutionenhanced dispersion by supercritical fluids.

\section{Acknowledgment}

This work was sponsored by the Interdisciplinary Program of Shanghai Jiao Tong University (project number YG2016QN08).

\section{Disclosure}

The authors report no conflicts of interest in this work.

\section{References}

1. Gao J, Chen G, He H-Q, et al. Therapeutic effects of breviscapine in cardiovascular diseases: a review. Front Pharmacol. 2017;8:289-302. doi:10.3389/fphar.2017.00289

2. Liu Z, Okeke C-I, Zhang L, et al. Mixed polyethylene glycol-modified breviscapine-loaded solid lipid nanoparticles for improved brain bioavailability: preparation, characterization, and in vivo cerebral microdialysis evaluation in adult Sprague dawley rats. AAPS PharmSciTech. 2014;15(2):483-496. doi:10.1208/s12249014-0080-4

3. Min W, Zhang W, Song J, et al. Effect of breviscapine on recovery of viable myocardium and left ventricular remodeling in chronic total occlusion patients after revascularization: rationale and design for a randomized controlled trial. Med Sci Mon Int Med J Exp Clin Res. 2018;24:4602-4609.

4. Wang H, Zhang G-X, Ma X-Q, et al. Enhanced encapsulation and bioavailability of breviscapine in PLGA microparticles by nanocrystal and water-soluble polymer template techniques. Eur J Pharm Biopharm. 2017;115:177-185. doi:10.1016/j.ejpb.2017.02.021

5. Cong W, Shen L, Xu D, et al. Solid dispersion tablets of breviscapine with polyvinylpyrrolidone K30 for improved dissolution and bioavailability to commercial breviscapine tablets in beagle dogs. Eur J Drug Metab Pharmacokinet. 2014;39(3):203-210. doi:10.1007/ s13318-013-0150-0

6. Ju A, Li Y, Qu Z, et al. Impact of the herbal breviscapine on the pharmacokinetics of simvastatin in rats: the involvement of CYP3A4. Drug Res. 2017;67(05):271-274. doi:10.1055/s-0042-118170

7. Shao D-Z, Wang C-K, Hwang H-J, et al. Abstracts: comparison of hydration, tyrosinase resistance, and antioxidant activation in three kinds of pearl powders. Int $J$ Cosmet Sci. 2010;32(5):396. doi:10.1111/j.1468-2494.2010.00609_5.x

8. Onodera R, Hayashi T, Nakamura T, et al. Preparation of silymarin nanocrystals using a novel high pressure crystallization technique and evaluation of its dissolution and absorption properties. Asian J Pharm Sci. 2016;11(1):211-212. doi:10.1016/j.ajps.2015.11.031

9. Kazi M, Al-qarni H, Alanazi FK. Development of oral solid self-emulsifying lipid formulations of risperidone with improved, in vitro, dissolution and digestion. Eur $J$ Pharm Biopharm. 2017;114:239-249.

10. Liu X, Zhou L, Zhang F. Reactive melt extrusion to improve the dissolution performance and physical stability of naproxen amorphous solid dispersions. Mol Pharm. 2017;14(3):658-674. doi:10.1021/acs.molpharmaceut.6b00960

11. Wang Y, Zhao Q-F, Han N, et al. Mesoporous silica nanoparticles in drug delivery and biomedical applications. Nanomedicine. 2015;11 (2):313-327. doi:10.1016/j.nano.2014.09.014

12. Chang J-H, Tsai P-H, Wang K-Y, et al. Generation of functional dopaminergic neurons from reprogramming fibroblasts by nonviral-based mesoporous silica nanoparticles. Sci Rep. 2018;8 (1):11-23. doi:10.1038/s41598-017-18324-8

13. Song Y, Li Y-H, Xu Q, et al. Mesoporous silica nanoparticles for stimuli-responsive controlled drug delivery: advances, challenges, and outlook. Int J Nanomedicine. 2017;12:87-110. doi:10.2147/IJN. S117495 
14. Xie X, Li F, Zhang H. EpCAM aptamer-functionalized mesoporous silica nanoparticles for efficient colon cancer cell-targeted drug delivery. Eur J Pharm Sci. 2016;83:28-35. doi:10.1016/j.ejps.2015.12.014

15. Zhang Y-Z, Zhang H, Che E, et al. Development of novel mesoporous nanomatrix-supported lipid bilayers for oral sustained delivery of the water-insoluble drug, lovastatin. Colloids Surf B Biointerfaces. 2015;128:77-85. doi:10.1016/j.colsurfb.2015.02.021

16. He X, Zhao Y, He D, et al. ATP-responsive controlled release system using aptamer-functionalized mesoporous silica nanoparticles. Langmuir. 2012;28(35):12909-12915. doi:10.1021/la302767b

17. Genina N, Hadi B, Löbmann K. Hot melt extrusion as solvent-free technique for a continuous manufacturing of drug-loaded mesoporous silica. J Pharm Sci. 2018;107(1):149-155. doi:10.1016/j. xphs.2017.05.039

18. Li Z, Zhang Y, Zhang K, et al. Biotinylated-lipid bilayer coated mesoporous silica nanoparticles for improving the bioavailability and anti-leukaemia activity of Tanshinone IIA. Artif Cells Nanomed Biotechnol. 2018;46:1-10. doi:10.1080/21691401.2018.1431651

19. Gignone A, Manna L, Ronchetti S, et al. Incorporation of clotrimazole in Ordered Mesoporous Silica by supercritical CO2. Microporous Mesoporous Mater. 2014;200:291-296. doi:10.1016/j. micromeso.2014.05.031

20. Aiello R, Cavallaro G, Giammona G, et al. Mesoporous silicate as matrix for drug delivery systems of non-steroidal antinflammatory drugs. Stud Surf Sci Catal. 2002;142(02):1165-1172.

21. Span R, Wagner W. A new equation of state for carbon dioxide covering the fluid region from the triple-point temperature to 1100 $\mathrm{K}$ at pressures up to $800 \mathrm{MPa}$. J Phys Chem Ref Data. 1996;25 (6):1509-1596. doi:10.1063/1.555991

22. Yin X, Daintree L-S, Ding S, et al. Itraconazole solid dispersion prepared by a supercritical fluid technique: preparation, in vitro characterization, and bioavailability in beagle dogs. Drug Des Dev Ther. 2015;9:4387.

23. Kankala R-K, Zhang Y-S, Wang S-B, et al. Supercritical fluid technology: an emphasis on drug delivery and related biomedical applications. Adv Healthc Mater. 2017;6(16):1-31.

24. Bouledjouidja A, Masmoudi Y, Li Y, et al. Supercritical impregnation and optical characterization of loaded foldable intraocular lenses using supercritical fluids. J Cataract Refract Surg. 2017;43 (10):1343-1349. doi:10.1016/j.jcrs.2017.07.033

25. Wang H-B, Yang -F-F, Gai X-M, et al. A pH-independent instantaneous release of flurbiprofen: a study of the preparation of complexes, their characterization and in vitro/vivo evaluation. Drug Dev Ind Pharm. 2017;43(9):1-34. doi:10.1080/03639045.2017.1318908

26. Oliveira G-E, Pinto J-F. Evaluation of the Potential Use of Laminar Extrudates on Stabilizing Micronized Coumarin Particles by Supercritical Fluids (RESS)-Study of Different RESS Processing Variables and Mode of Operation. AAPS PharmSciTech. 2017;18 (7):1-16. doi:10.1208/s12249-017-0760-y

27. Vo D-T, Saravana P-S, Woo H-C, et al. Fucoxanthin-rich oil encapsulation using biodegradable polyethylene glycol and particles from gas-saturated solutions technique. J CO2 Util. 2018;26:359-369. doi:10.1016/j.jcou.2018.05.019

28. Kurniawansyah F, Quachie L, Mammucari R, et al. Improving the dissolution properties of curcumin using dense gas antisolvent technology. Int J Pharm. 2017;521(1-2):239-248. doi:10.1016/j. ijpharm.2017.02.018

29. Villanueva-bermejo D, Zahran F, Troconis D, et al. Selective precipitation of phenolic compounds from Achillea millefolium L. extracts by supercritical anti-solvent technique. $J$ Supercrit Fluids. 2017;120:52-58. doi:10.1016/j.supflu.2016.10.011

30. Kaga K, Honda M, Adachi T, et al. Nanoparticle formation of PVP/ astaxanthin inclusion complex by solution-enhanced dispersion by supercritical fluids (SEDS): effect of PVP and astaxanthin Z isomer content. J Supercrit Fluids. 2018;136:44-51. doi:10.1016/j. supflu.2018.02.008
31. Obaidat R-M, Tashtoush B-M, Awad -A-A, et al. Using supercritical fluid technology (SFT) in preparation of tacrolimus solid dispersions. AAPS PharmSciTech. 2016;18(2):1-13. doi:10.1208/s12249-0160685-x

32. Xie M, Fan D, Yi L, et al. Supercritical carbon dioxide-developed silk fibroin nanoplatform for smart colon cancer therapy. Int J Nanomedicine. 2017;12:7751-7761. doi:10.2147/IJN.S145012

33. Tripathi N, Yamashita M, Uchida $T$, et al. Observations on size confinement effect in B-C-N nanoparticles embedded in mesoporous silica channels. Appl Phys Lett. 2014;105(1):7202-R43.

34. Gang Y, Zhe L, Shao Q, et al. Measurement and correlation study of silymarin solubility in supercritical carbon dioxide with and without a cosolvent using semi-empirical models and back-propagation artificial neural networks. Asian J Pharm Sci. 2017;12:456-463. doi:10.1016/j.ajps.2017.04.004

35. Hu L, Sun H, Zhao Q, et al. Multilayer encapsulated mesoporous silica nanospheres as an oral sustained drug delivery system for the poorly water-soluble drug felodipine. Mater Sci Eng C. 2015;47:313-324. doi:10.1016/j.msec.2014.10.067

36. Garay I, Pochevillea A, Madariagaa L. Polymeric microparticles prepared bysupercritical antisolvent precipitation. Powder Technol. 2010;197:211-217. doi:10.1016/j.powtec.2009.09.015

37. Jin J-S, Ning -Y-Y, Hu K, et al. Solubility of p-nitroaniline in supercritical carbon dioxide with and without mixedcosolvents. J Chem Eng Data. 2013;58:1464-1469. doi:10.1021/je300987d

38. Nadia E, Ghoreishi S-M. Synthesis of 5-Fluorouracil nanoparticles via supercritical gas antisolvent process. $J$ Supercrit Fluids. 2013;84:205-210. doi:10.1016/j.supflu.2013.10.008

39. Li L-L, Liu T-L, Fu C-H, et al. Biodistribution, excretion, and toxicity of mesoporous silica nanoparticles after oral administration depend on their shape. Nanomed Nanotechnol Biol Med. 2015;11 (8):1915-1924. doi:10.1016/j.nano.2015.07.004

40. Jia L-J, Shen J-Y, Li Z-Y, et al. Successfully tailoring the pore size of mesoporous silica nanoparticles: exploitation of delivery systems for poorly water-soluble drugs. Int $J$ Pharm. 2012;439:81-91. doi:10.1016/j.ijpharm.2012.10.011

41. He Y, Liang S, Long M, et al. Mesoporous silica nanoparticles as potential carriers for enhanced drug solubility of paclitaxel. Mater Sci Eng C. 2017;78:12-17. doi:10.1016/j.msec.2017.04.049

42. Juan J-G, Paloma M-T, Vegas-sánchez M-C, et al. Changed crystallinity of mebendazole solid dispersion: improved anthelmintic activity. Int $J$ Pharm. 2011;403(1-2):23-28. doi:10.1016/j. ijpharm.2010.10.002

43. Ke K, Wei X-F, Bao R-Y, et al. Contribution of residual solvent to the nucleation and reinforcement of poly (vinylidene fluoride). Polym Test. 2014;34:78-84. doi:10.1016/j.polymertesting.2013.12. 012

44. Akbuga J, Gursoy A, Kendi E. The preparation and stability of fast release furosemide-PVP solid dispersion. Drug Dev Ind Pharm. 1988;14:1439-1464. doi:10.3109/03639048809151942

45. Horkovics-kovats S. Dissolution and coarsening of polydisperse, polymorph drug particles liberated from a disintegrating finished dosage form: theoreticalconsiderations. Eur $J$ Pharm Sci. 2016;91:265-277. doi:10.1016/j.ejps.2016.05.003

46. $\mathrm{Wu} \mathrm{C}$-E. Increasing the oral bioavailability of poorly water-soluble carbamazepine using immediate-release pellets supported on SBA-15 mesoporous silica. Int $J$ Nanomedicine. 2012;7:5807-5818. doi:10.2147/IJN.S37650

47. Xiong F, Xiong C, Yao J, et al. Preparation, characterization and evaluation of breviscapine lipid emulsions coated with monooleatePEG-COOH. Int $J$ Pharm. 2011;421(2):275-282. doi:10.1016/j. ijpharm.2011.10.008

48. Lv W, Guo J, Li J, et al. Distribution of liposomal breviscapine in brain following intravenous injection in rats. Int J Pharm. 2005;306 (1-2):99-106. doi:10.1016/j.ijpharm.2005.09.012 


\section{Publish your work in this journal}

The International Journal of Nanomedicine is an international, peerreviewed journal focusing on the application of nanotechnology in diagnostics, therapeutics, and drug delivery systems throughout the biomedical field. This journal is indexed on PubMed Central, MedLine, CAS, SciSearch ${ }^{\mathbb{R}}$, Current Contents ${ }^{\mathbb{B}} /$ Clinical Medicine,
Journal Citation Reports/Science Edition, EMBase, Scopus and the Elsevier Bibliographic databases. The manuscript management system is completely online and includes a very quick and fair peer-review system, which is all easy to use. Visit http://www.dovepress.com/ testimonials.php to read real quotes from published authors.

Submit your manuscript here: https://www.dovepress.com/international-journal-of-nanomedicine-journal 